DOI: https://doi.org/10.46296/yc.v5i8edespmar.0098

\title{
COVID-19: UNA MIRADA EN PEDAGOGÍA VIRTUAL EN EL CONTEXTO UNIVERSITARIO
}

\section{COVID-19: A LOOK AT VIRTUAL PEDAGOGY IN THE UNIVERSITY CONTEXT}

\author{
Cedeño-Barcia Lizandro Agustín ${ }^{1 *}$; Flores-Tena María José ${ }^{2}$; Cedeño-Barcia María \\ Monserrate ${ }^{3}$ \\ ${ }^{1}$ Docente de la Universidad Técnica de Manabí, UTM. Portoviejo - Ecuador. \\ ${ }^{2}$ Docente de la Universidad Autónoma de Madrid. Madrid - España. \\ ${ }^{3}$ Docente de la Universidad Técnica de Manabí, UTM. Portoviejo - Ecuador.
}

*Correo: lizandrocedenobarcia@gmail.com

\begin{abstract}
Resumen
El objetivo de la investigación es identificar cuáles han sido las principales herramientas pedagógicas que se implementaron dentro de las aulas virtuales de una institución de educación superior de Ecuador. Se aplica una metodología cuantitativa con un enfoque dinámico; para desarrollar el levantamiento de datos se desarrolló una batería en google forms que destaca los aspectos de: estrategias utilizadas, recursos y la forma de ingreso de los estudiantes para ejecutar las tareas proporcionadas por los docentes. Los resultados indican que la implementación de la modalidad virtual ha coexistido de manera positiva, llegando a compensar la continuidad de los estudios, y que los alumnos adoptaron una actitud efectiva. Como parte de una de las conclusiones, se puede empatar que la pandemia producida por el COVID-19, cambio la vida de los educandos, de las universidades, siendo la educación una de las más afectadas en donde han tenido que restructurar un sistema presencial a uno sincrónico y asincrónico.
\end{abstract}

Palabras claves: COVID-19, Pedagogía virtual, Plataforma virtual, herramientas pedagógicas.

\begin{abstract}
The objective of the research is to identify the main pedagogical tools implemented in the virtual classrooms of a higher education institution in Ecuador. A quantitative methodology with a dynamic approach is applied; to develop the data collection a battery was developed in google forms that highlights the aspects of: strategies used, resources and the form of entry of students to execute the tasks provided by teachers. The results indicate that the implementation of the virtual modality has coexisted in a positive way, compensating the continuity of studies, and that the students adopted an effective attitude. As part of one of the conclusions, it can be said that the pandemic produced by COVID-19 changed the lives of students and universities, with education being one of the most affected, where they have had to restructure a face-to-face system to a synchronous and asynchronous one.
\end{abstract}

Keywords: COVID-19, Virtual pedagogy, Virtual platform, pedagogical tools.

Información del manuscrito:

Fecha de recepción: 18 de enero de 2021.

Fecha de aceptación: 19 de marzo de 2021.

Fecha de publicación: 29 de marzo de 2021 


\section{Introducción}

El COVID-19, ha sido una de las pandemias del siglo XXI, que ha dejado muchas secuelas en los diferentes ámbitos personales, esta problemática afecto las áreas educativas, económicas, laborales, de salud y otras áreas que sin duda son fundamentales para el ser humano.

En correlación con lo expuesto anterior me, se examina cono se han venido manifestando los efectos retrospectivos sobre la pandemia, y a su vez que significa el Coronavirus (COVID-19), para la Organización Mundial de la Salud (OMS, 2020), indica que los coronavirus son una extensa familia de virus que pueden causar enfermedades tanto en animales como en humanos.

Simultáneamente el Director General de la Organización Mundial de la Salud (OMS), el doctor Tedros Adhanom Ghebreyesus, anunció el 11 de marzo de 2020 que la nueva enfermedad por el coronavirus 2019 (COVID-19) puede caracterizarse como una pandemia. La caracterización de pandemia significa que la epidemia se ha extendido por varios países, continentes o todo el mundo, y que afecta a un gran número de personas (OPS, 2020).

Para contrastar los efectos del COVID-19, en el margen social y sus efectos se los pueden evidenciar de manera inscripta en los informes presentados por la Comisión Económica para América Latina y el Caribe (CEPAL), organismo de examinar diferentes estudios y levantamientos sobre las desiguales y problemáticas que ocurren América latina. En la figura 1 , se puede observar varios aspectos sobre la emergencia, que dan a conocer una perspectiva sobre los disparejos grados de afectación que han tenido a raíz de la pandemia.

Desde otro aspecto el informe que presenta la misma organización CEPAL en donde explica que los primeros casos de COVID-19 se registraron en áreas urbanas, que se presentó una panorámica con mayor riesgo en las muertes a los pobres y vulnerables, como pulmonares, cardiovasculares y diabetes, la imposibilidad de 
estudiar de trabajar y así mismo los trabajos informales.

En cuanto a lo relacionado en los efectos sociales, un ítem que se mide es la problemática sobre la sociedad, es el cambio de sus actividades y el verse en una permutación de los nuevos procesos de adaptación (...) Las propiedades fundamentales de esta pandemia, y su comportamiento e inicio y dispersión a nivel planetario son razones indiscutibles de carácter complejo (Díaz, 2020, p.500).

Figura 1. Impacto Social

\section{Impacto Social}

- La caída económica agregará 12 millones de personas a las filas del desempleo (37.7m) y llevará a 28.7 millones de personas adicionales a la pobreza $(215 \mathrm{~m})$ y a 15.9 millones de personas a la pobreza extrema $(87 \mathrm{~m})$.

- La CEPAL propone un ingreso básico de emergencia equivalente a una línea de pobreza durante seis meses para toda la población que vive en la pobreza en 2020 (215 millones, el 34,7\% de la población).
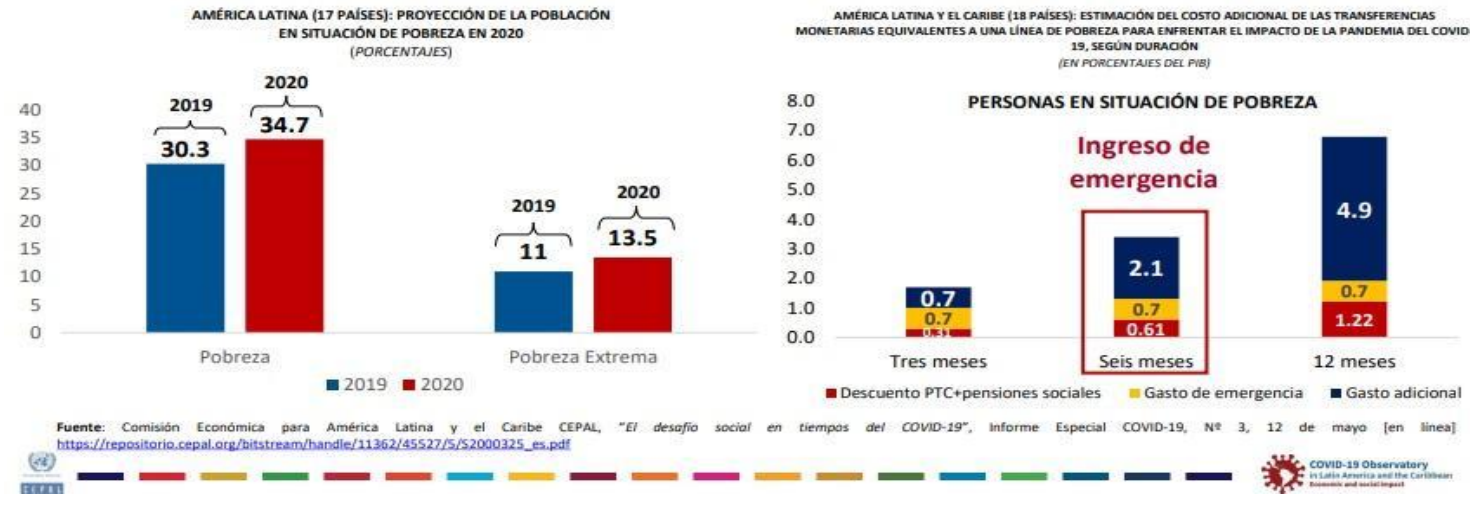

Fuente: CEPAL.

El análisis crítico referente a este proceso es identificar cuáles fueron las mayores esferas sociales que lograron ser afectadas a nivel macro, meso, micro. En este sentido el examinar el contexto universitario es fundamental, como espacio que genera la percepción y formación de nuevos profesionales, además de conocer cómo se dieron cuenta en los grados de acciones sobre la pandemia, y que permiten establecer qué estrategias tomaron las universidades para llevar a cabo la metodología de aprendizaje durante la pandemia.

Así mismo, la pandemia ya ha tenido un enorme impacto en la educación con el cierre de las escuelas en casi todos los lugares del planeta, en lo que representa la crisis simultánea más importante que han sufrido todos los sistemas educativos del mundo en nuestra época. El daño será aún más grave 
a medida que la emergencia de salud se traslade a la economía y provoque una profunda recesión mundial (Vicentini, 2020).

Del mismo modo que la pandemia ha causado un serie de problemáticas en el contexto de educación, su interpretación ante la respuestas del sistema educativa, esta llegan a ser examinadas de manera efectivas y su manifestación desde un orden crítico (...) Se debe reconocer que en el sector de educación superior tampoco se encontraron preparado para una disrupción como la que ha traído consigo la pandemia del COVID-19, en los presentes como son los cierres, como medidas para contener la pandemia, ha llevado a un despliegue acelerado de soluciones en la educación superior (Francesc, 2020).

Desde un punto más amplio, las universidades que habían iniciado una transición a la digitalización antes de la pandemia y que contaban con una infraestructura tecnología ya tenían cierta experiencia en el desarrollo de una cultura digital, con estudiantes y docentes adaptados a mecanismos como trámites digitalizados y cursos presenciales dictados en un formato híbrido y con el contenido curricular en línea (Banco Interamericano de Desarrollo, 2020, p.4).

La problemática de esta situación, no solo tiende en saber cuántas universidades usaban la modalidad virtual, sino en conocer cómo eran los recursos tecnológicos que tienen las universidades dentro del contexto de educación, la formación de un currículum que logre ser establecido dentro del argumento de los requerimientos educativos.

No obstante, las fórmulas tradicionales de educación a distancia, es decir, aquellas en las que el profesor sigue impartiendo una clase ordinaria que es retransmitida en directo y que puede ser recuperada en diferido, parecen ser las más apreciadas por los estudiantes porque son las que mejor reproducen la dinámica a la que están acostumbrados (Unesco/lesalc, 2020).

Para Buxarrais (2011) Las nuevas tecnologías, entendidas como los dispositivos digitales que se pueden conectar con un ordenador o con internet, son, probablemente, las herramientas más potentes, 
versátiles y ubicuas que la sociedad haya conocido.

La idea de la educación virtual se ha venido consolidando alrededor de las Tecnologías de Información y Comunicación, asociada a comprensiones que permiten el reconocimiento de lo virtual como simulación de la realidad del aula, los contenidos, el currículo, el que enseña, los que aprenden, en conjunto la educación escenificada en entornos digitales (Rozo, 2010, p.34).

Los entornos virtuales de aprendizajes en la educación se basan en el principio de aprendizaje colaborativo donde se permite a los alumnos realizar sus aportes y expresar sus inquietudes en los foros, como parte de una comunicación de interacción de estas aulas virtuales, que ahora se utilizan como mecanismos positivos para la impartición de las clases (Hiraldo, 2013).

Por una parte la conectividad y los mecanismos que se deben adaptar para el proceso de aprendizaje y enseñanza virtual logran considerarse necesarios para adaptar el esquema de la brecha digital, para el autor Silva (2017) es ello que los Learning Management Systems (LMS), permiten el diseño de entornos virtuales de aprendizajes (EVA), espacios formativos que para innovar en la docencia requieren utilizar metodologías centradas en el alumno, cambio de roles en docentes y estudiantes, incorporar las TIC para colaborar, compartir y distribuir conocimiento en red. Especialmente a nivel universitario.

Ahora bien, se puede conceptualizar que una plataforma virtual de aprendizaje no es más que una aplicación informática diseñada para facilitar la comunicación pedagógica entre los participantes en este tipo de proceso. Es un software que proporciona la logística necesaria para llevar a cabo la formación online, permite la creación, almacenamiento y publicación de objetos de aprendizaje. (Rodríguez, 2018).

El identificar las principales herramientas pedagógicas que se implementaron en las aulas virtuales de la Universidad Técnica de Manabí, llegan a ser un objetivo planteado, a raíz de la pandemia por COVID-19 como parte del área 
docente el indagar como los sistemas de educación han realizado métodos capaces de remplazar al sistema presencial a un virtual, puede llegar a ser un primer acercamiento para la producción de nuevas metodologías, que consistan en satisfacer las necesidades que en cierto contexto son de relevancia para estudios continuos sobre esta temática del área de educación.

\section{Objetivo}

Identificar las herramientas pedagógicas implementadas en las aulas virtuales de la Universidad Técnica de Manabí durante el periodo de pandemia por COVID19.

\section{Metodología}

La investigación pretendió el identificar cuáles han sido las principales herramientas pedagógicas que se implementaron en las aulas virtuales de la Universidad Técnica de Manabí, para aquello se aplicó una metodología cuantitativa con un enfoque dinámico, para desarrollar este proceso se describe a continuación su ejecución.

\section{Métodos y técnicas de la investigación:}

Para desarrollar la investigación se aplicó una encuesta mediante la plataforma de google forms, de los cuales se midieron los siguientes aspectos: virtualidad por la pandemia, actividades diseñadas en el aula virtual, dificultad de ingreso. Para desarrollar el proceso de la investigación los resultados se establecieron mediante la representación de tablas y gráficos.

\section{Población y muestra:}

Para seleccionar la población se identificó que sean estudiantes que cursen el semestre de mayo 2020octubre del 2020, dentro de la Universidad Técnica de Manabí. Al momento que se seleccionó la muestra se tuvo un alcance de 100 alumnos que lograron representar un primer acercamiento a los datos sobre en qué estado se encuentra la modalidad virtual, y que características existen dentro de las aulas virtuales. La investigación se planteó con el objeto de identificar cuáles son las principales 
herramientas didácticas que han sido implementadas dentro de la plataforma de la universidad y a que raíz de la pandemia del COVID 19 este es un estudio como primer acercamiento sobre el desarrollo de la virtualidad.

\section{Análisis de resultados}

Para realizar el levantamiento de datos se desarrolló una encuesta estructurada que fue aplicada a 100 estudiantes que cursan diferentes materias dentro de la Universidad Técnica de Manabí, con el fin de conocer en qué estado se encuentra el desarrollo de la modalidad virtual.

A continuación, se describen los resultados obtenidos, esto fue dentro del primer trimestre de estudios que han cruzado los estudiantes.

Tabla 1. Virtualidad ante el COVID-19.

\begin{tabular}{|c|c|c|c|}
\hline ÍTEMS & CANTIDAD & $\%$ & TOTAL \\
\hline Muy positivo & 10 & $10 \%$ & 10 \\
\hline Positivo & 50 & $50 \%$ & 50 \\
\hline Básico & 26 & $26 \%$ & 26 \\
\hline Regular & 14 & $14 \%$ & 14 \\
\hline \multicolumn{2}{|r|}{} & & \multirow{2}{*}{100} \\
\cline { 2 - 4 }
\end{tabular}

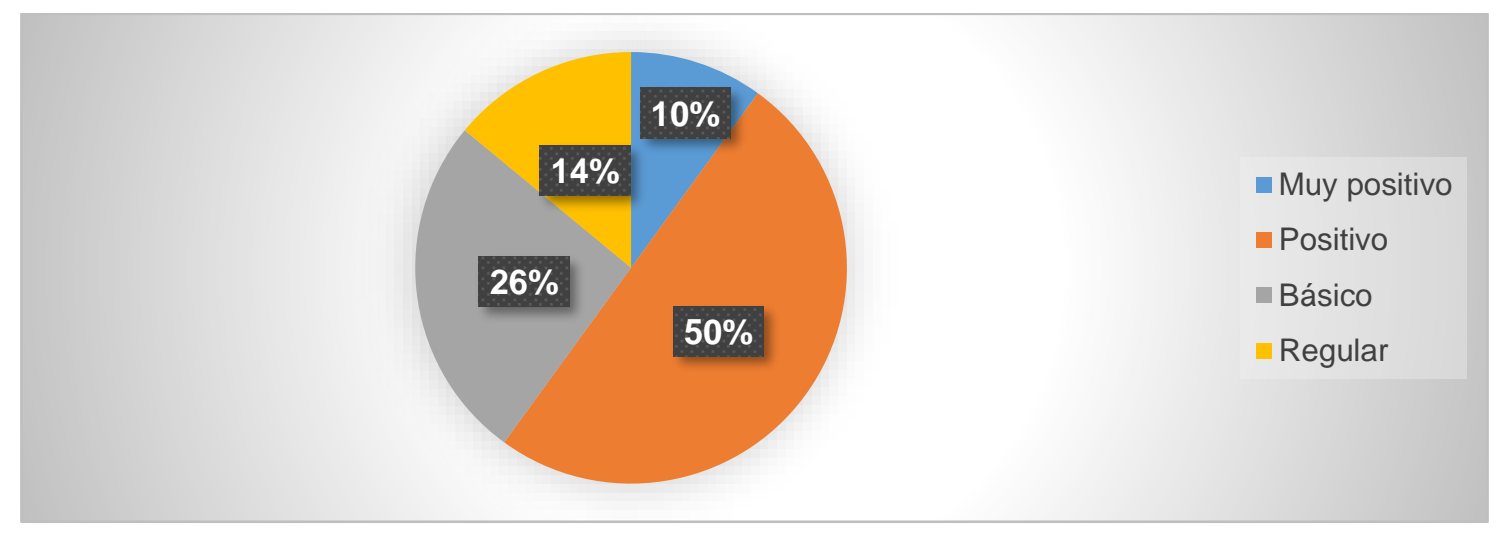

Fuente: Participantes de la investigación.

Elaboración: Propia.

Análisis e interpretación:

Como se puede observar en la tabla 1 , los estudiantes responden que el estudio virtual a raíz de la pandemia por COVID-19, se ha formulado de manera positiva con un 50\% de satisfacción en la escala estadística del estudio, esto aclara que el 
implementar una modalidad puede ser un efecto asertivo, y que la universidad aporta de manera segura, y al cuidado de la salud de los estudiantes. Un $14 \%$ de los participantes indican que la modalidad virtual es de forma regular, esto se puede dar a raíz que los estudiantes que cursan sus estudios debieron acoplarse de manera no presencial, pero se puede manejar de manera adaptable por qué lo identificaron de esta forma. En este sentido se torna a lo que el autor Durán (2015) indica, que la educación virtual posee características que la diferencian en gran medida de la educación presencial. Primero, existe una mayor autonomía e independencia que disfruta el alumnado para el desarrollo de su proceso de aprendizaje, siendo el estudiante quien marca su ritmo de trabajo.

Tabla 2. Actividades en el aula virtual

\begin{tabular}{|c|c|c|c|}
\hline ÍTEMS & CANTIDAD & $\%$ & TOTAL \\
\hline Especificas & 30 & $30 \%$ & 30 \\
\hline Dinámicas & 40 & $40 \%$ & 40 \\
\hline Significativas & 24 & $24 \%$ & 24 \\
\hline Muy poco significativas & 6 & $6 \%$ & 6 \\
\hline \multicolumn{2}{|r|}{} & 100 \\
\hline
\end{tabular}

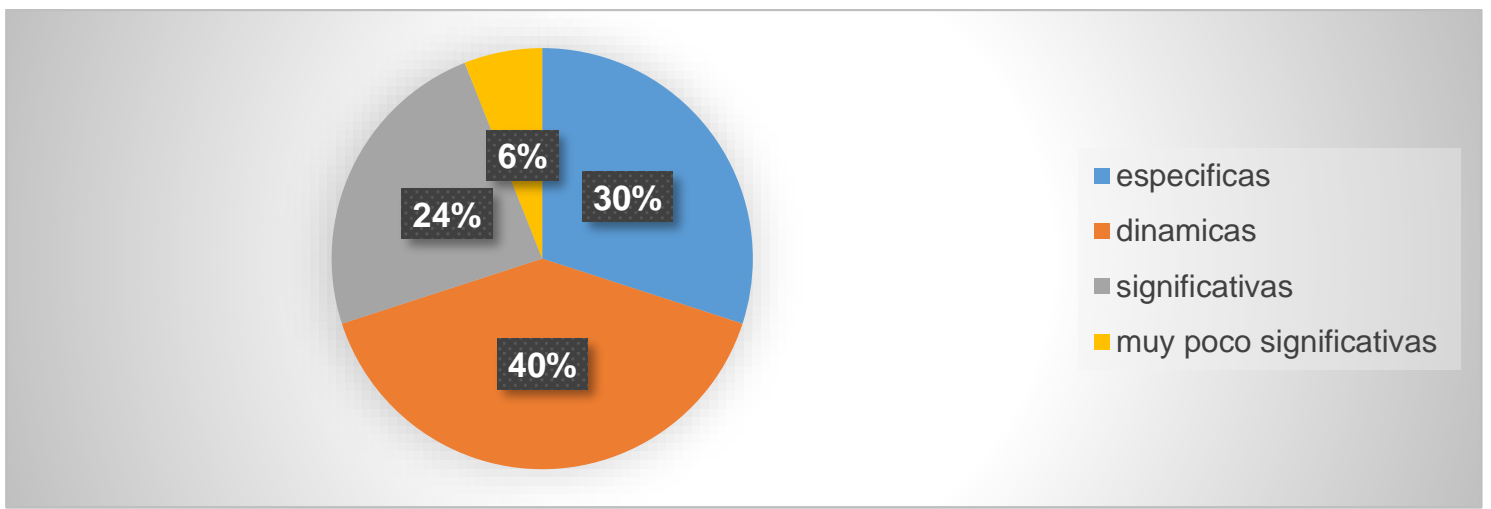

Fuente: Participantes de la investigación.

Elaboración: Propia.

Análisis e interpretación:

A continuación se puede observar que en la tabla 2, que las actividades que se han desarrollado dentro del sistema virtual, permitió identificar que son de manera dinámicas, que producen un sistema aceptable con los 
estudiantes en su proceso de formación, esto se encuentra validado en el $40 \%$ de la muestra encuestada, el $30 \%$ indicaron que las programaciones de las clases son específicas, esto se da quizás al tipo de materia y el recurso pedagógico con él cuenta el docente para el desarrollo del docente. El 24\% identificaron que las actividades son significativas, esto a su vez puede relacionarse con el contexto del área de estudio ya que son proporcionadas como materias de transversalidad y formación universitaria. Si se hiciera uso de estas tecnologías, los alumnos podrían incrementar su rendimiento académico aprovechando los elementos y recursos que ofrece esta tecnología; además las aulas digitales en la educación superior apoyan a combatir el rezago educativo, por lo tanto, sin embargo pueden corregir sus deficiencias de aprendizaje mediante el uso de aulas virtuales; en consecuencia las escuelas de educación superior deben implementar capacitación sobre el uso de aulas virtuales a profesores y alumnos. (Monroy, 2018).

Tabla 3. Tipo de actividad en el aula virtual.

\begin{tabular}{|c|c|c|c|}
\hline ÍTEMS & CANTIDAD & $\%$ & TOTAL \\
\hline Videos & 40 & $40 \%$ & 40 \\
\hline Explicaciones interactivas & 33 & $33 \%$ & 33 \\
\hline Videos detallados & 14 & $14 \%$ & 14 \\
\hline Juegos & 13 & $13 \%$ & 13 \\
\hline \multicolumn{2}{|r}{} & 100 \\
\cline { 4 - 4 }
\end{tabular}

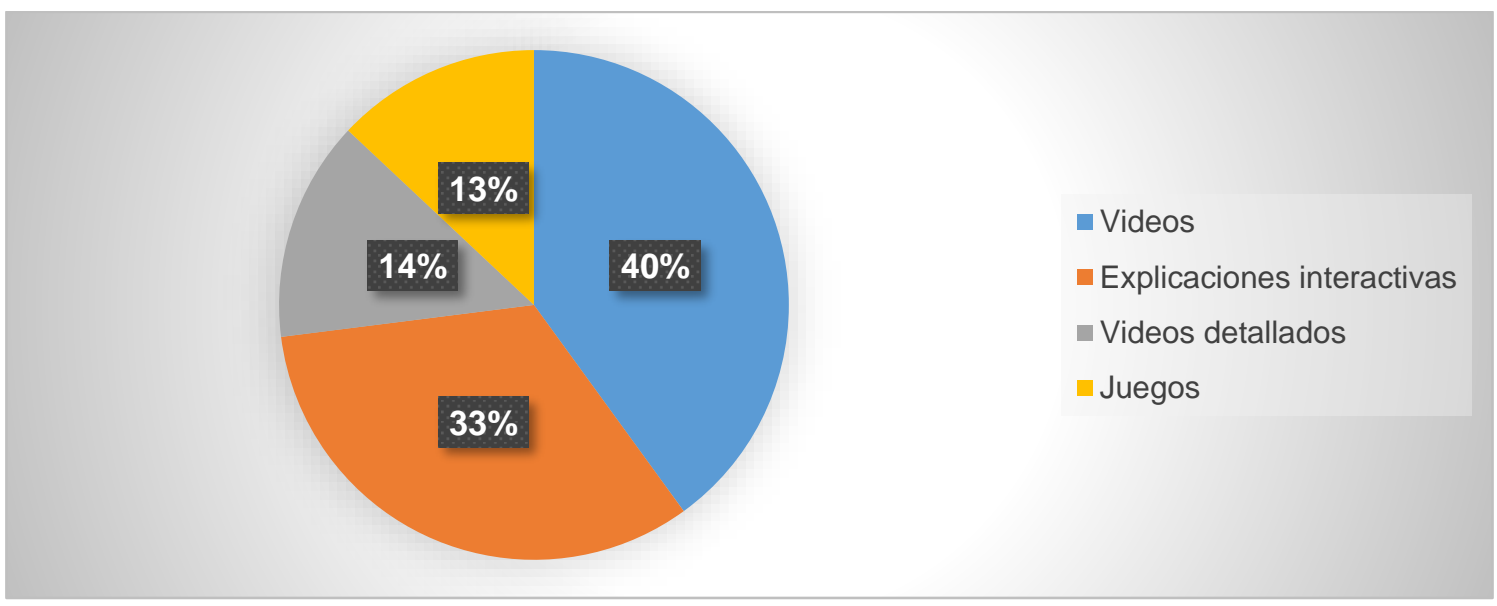

Fuente: Participantes de la investigación.

Elaboración: Propia. 
Análisis e interpretación:

Como se puede visualizar dentro de la tabla 3, los participantes de la investigación un $40 \%$ indicaron que los recursos y actividades que están facilitadas en el aula son videos, como tal objeto estos funcionan como parte del objeto visual y del mecanismo de propiedad que este brinda al facilitar contenidos interactivos, como lo reafirma el autor Sandoval (2018), el uso de vídeos teóricos y explicativos aclaran conceptos, definiciones y enunciados.

La mayoría de los estudiantes piensan que es una buena herramienta de aprendizaje tener vídeos sobre las partes más importantes de la clase, especialmente en la resolución de ejercicios, gran parte de ellos le dedicaría un tiempo mayor a 10 minutos para visualizar $y$ complementar su formación académica. Un 33\% determinó que otra de las formas que han diseñado dentro del aula para el aprendizaje son las explicaciones interactivas, es donde el docente logra desarrollar una estrategia de comunicación efectiva, valorizando el conocimiento y la información asincrónica de manera que produzca una acción positiva de la misma.

El $13 \%$ de la muestra afirmó que existen juegos lúdicos dentro del aula, dicen que el beneficio de los juegos es que les permite recordar de forma interactiva conocimientos ya previamente adquiridos a través de la lectura. Si bien, muchos pedagogos son radicales con el tema del juego en el ámbito educativo, otros tantos, miran con interés la posibilidad de dinamizar el proceso de formación a través de estrategias educativas basadas en el juego, rescatan que en los entornos virtuales se requiere de la motivación y persuasión para orientar al estudiante, tratar de que él cumpla con sus actividades (Melo, 2017).

Tabla 4. Dificultades en el aula.

\begin{tabular}{|c|c|c|c|}
\hline ÍTEMS & CANTIDAD & $\%$ & TOTAL \\
\hline No & 70 & $70 \%$ & 70 \\
\hline Pocas veces & 24 & $24 \%$ & 24 \\
\hline
\end{tabular}




\begin{tabular}{|c|c|c|c|} 
Muchas veces & 6 & $6 \%$ & 6 \\
\hline
\end{tabular}

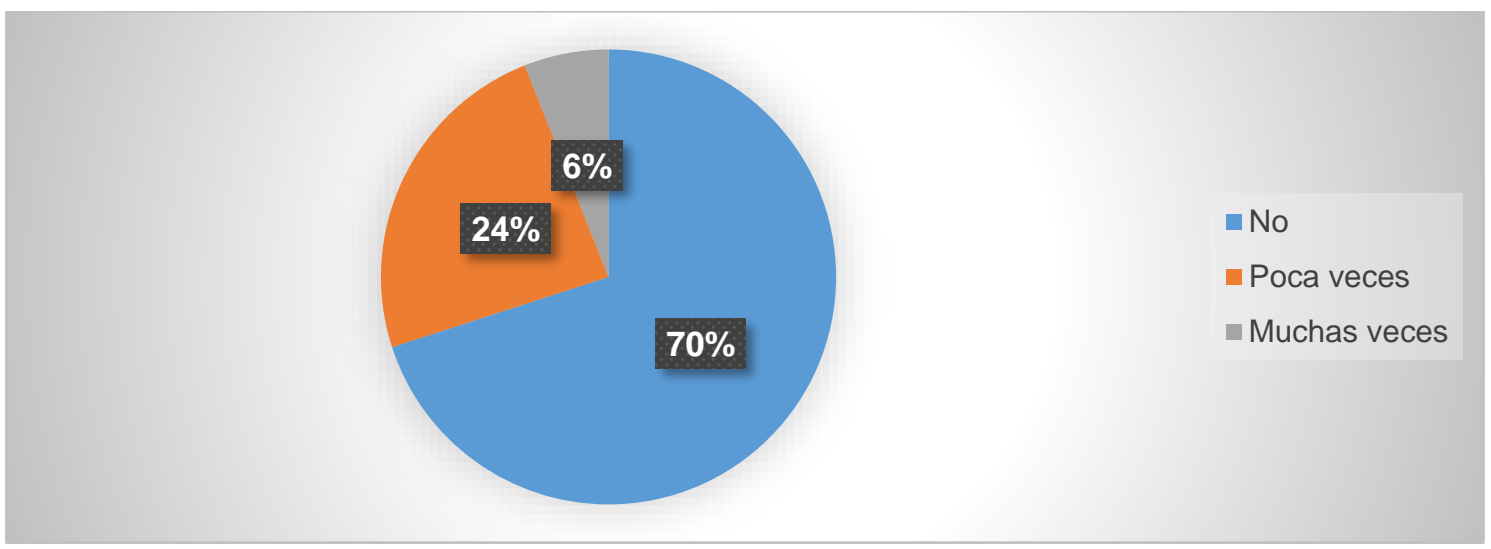

Fuente: Participantes de la investigación.

Elaboración: Propia.

Análisis e interpretación:

Dentro del contexto de ingreso, y de la realización de las diferentes actividades propuestas en el aula, un $70 \%$ de los estudiantes no tienen problema con el desarrollo de las mismas, esto significa que modalidad virtual ante la pandemia logra ser una de las medidas de mayor asertividad, que se logra mediante las estructuras lúdicas y correlaciónales entre la interacción de conocimientos, un $24 \%$ asegura que pocas veces tienen dificultades en esto, a su vez se puede caracterizar porque los grados de interpretación logran ser de manera compleja a explicaciones que son puntos exactos dentro de la actividad a ejecutarse. En efecto, las instituciones deben ajustar la convergencia entre la educación tradicional y la implementación de las nuevas tecnologías, si estas son usadas de forma adecuada por los docentes, pues de lo contrario pueden generar grandes debilidades en el proceso educativo dentro y fuera de los salones de clases (Sierra, 2018, p. 33).

\section{Conclusiones}

El COVID-19, ha sido una de las pandemias del siglo $\mathrm{XXI}$, que ha logrado afectar de manera mundial los diferentes contextos por lo que los seres humanos han estado empleados a ser, en cierto aspecto la emergencia sanitaria ha cambiado el estilo de vida, proporcionando mecanismo de adaptabilidad, y siendo el recurso 
tecnológico uno de los mayores soportes para la sociedad. El incrementar métodos de manera sincrónica y asincrónica ha sido un producto significativo para los diferentes espacios, entre ellos lo sociales, laborales, educativos y de actividad de producción que son factores de beneficios para el ser humano.

Las universidades como parte de los sistemas se han visto obligadas a generar el estudio virtual, han tenido una gran responsabilidad en prevenir contagios y resguardar la seguridad sanitaria de su comunidad educativa, siendo esta una de las primeras entidades educativas en gestionar mecanismos que les permitan tener un acercamiento para el avances dentro de los saberes con sus estudiantes y a su vez logren un mejor alcance para que estos puedan mantener un vínculo entre los procesos educativos y varios aspectos en dar continuidad a las programaciones dentro de sus componentes de trabajo.

El aprendizaje virtual como tal, ejecuta una serie de procesos que involucran la aplicación de métodos y técnicas que lleguen a ser capaces de satisfacer y consolidar los conocimientos que son ejecutados de manera presencial con el docente, y asimilados por el estudiante, las tic como tal, son un recurso capaz de acaparar un sistema sincrónico y asincrónico que a la vez consoliden a un currículum formativo y que sea capaz de establecer herramientas útiles de satisfacción dentro de la enseñanza.

De acuerdo a los resultados presentados en las tablas 1, 2,3 y 4, se cumple un primer acercamiento hacia las condiciones y relaciones de interpretación que existen para aplicar didácticas dentro de la construcción de la virtualidad, si bien es cierto las instituciones de educación superior (IES), cuenta con materias presenciales que son parte formativa en una profesión, se acoplan al sistema virtual para proporcionar actividades capaces de cumplir con los requerimientos, que el alumno tenga la facilidad de realizar el proceso a ejecutarse.

En relación con la tabla 3 , un dato muy importante es que el $13 \%$ de la muestra afirmó que existen juegos lúdicos dentro del aula, esto puede ser una de las formas de mayor 
asertividad para mejorar el aprendizaje de los estudiantes, es siendo una estrategia de factibilidad que fomenta el espacio de conocimiento a través de juegos que llegan a estar relacionados con lecturas previas.

\section{Bibliografía}

Banco Interamericano de Desarrollo. (2020). La educación superior en tiempos de Covid-19, aportes de la segunda reunión del dialogo virtual con rectores de Universidad líderes de América Latina. Recuperado de

https://publications.iadb.org/p ublications/spanish/document /La-educacion-superior-entiempos-de-COVID-19-

Aportes-de-la-Segunda-

Reunion-del-Di\%C3\%A1logoVirtual-con-Rectores-deUniversidades-Lideres-deAmerica-Latina.pdf

Buxarrais, M, \& Ovide, E. (2011). El impacto de las nuevas tecnologías en la educación en valores del siglo $\mathrm{XXI}$. Sinéctica, (37), 1-14. Recuperado de. http://www.scielo.org.mx/sciel o.php?script=sci_arttext\&pid= S1665109X2011000200002\&lng=es \&tlng=es.
CEPAL. (2020). Los Efectos Económicos y Sociales del COVID-19 en América Latina y el Caribe Legislando para la recuperación económica frente al COVID-19 Diálogo cooperativo entre las y los presidentes de los poderes legislativos de América Latina - ParlAmericas. (Documento PDF). Recuperado de https://www.cepal.org/sites/d efault/files/presentation/files/2 00605_final_presentacion_pa rlamericasv_alicia_barcena.p df

Díaz, J, Valdés, M. (2020). La pandemia de COVID 19 y sus implicaciones en la concepción, diseño e instrumentación didáctica de la educación médica superior cubana. MediSur, 18(3) .496506. Recuperado de: https://www.redalyc.org/articu lo.oa?id=1800/18006343702 2

Duran, R. (2015). La Educación Virtual Universitaria como medio para mejorar las competencias genéricas y los aprendizajes a través de buenas prácticas docentes. (Tesis doctoral). Universidad Politécnica de Cataluña. Barcelona, España. Recuperado de https://www.tdx.cat/bitstream/ handle/10803/397710/TRAD R1de1.pdf?sequence $=1$ \&isAll owed $=y$ 
Francesc, P. (2020). COVID-19 Y EDUCACIÓN SUPERIOR EN AMÉRICA LATINA Y EL CARIBE: EFECTOS, IMPACTOS $Y$ RECOMENDACIONES POLÍTICAS. Recuperado de https://www.fundacioncarolin a.es/wpcontent/uploads/2020/06/AC36.-2020.pdf

Hiraldo, R. (2013). Uso de los entornos virtuales de aprendizaje en la educación a distancia. Edutec. 2-14. Recuperado de https://www.uned.ac.cr/acade mica/edutec/memoria/ponenc ias/hiraldo_162.pdf

Monroy, A, Hernández, I, Jiménez, M. (2018). Aulas Digitales en la Educación Superior: Caso México.

Formación universitaria, 11 (5). 93-104. Recuperado de https://scielo.conicyt.cl/pdf/for muniv/v11n5/0718-5006formuniv-11-05-00093.pdf

OPS. (2020). Enfermedad por el Coronavirus (COVID-19). [Mensaje de un blog]. Recuperado de https://www.paho.org/es/tag/e nfermedad-por-coronaviruscovid-19

Organización Mundial de la Salud. (2020). Qué es un coronavirus? [Mensaje de un blog]. Recuperado de https://www.who.int/es/emerg encies/diseases/novel- coronavirus-2019/advice-for-

public/q-a-

coronaviruses?gclid=CjwKCA jwjLD4BRAiEiwAg5NBFnFQ WMOrR_zCBSWqSuctlafKZ $\mathrm{r}$ DfzqDAh1lvyEtBT8vV3awS3 5osgBoC-H4QAvD_BwE

Rodríguez, K, Pérez, J, Torres, G. (2018). Implementación de un entorno virtual como herramienta didáctica para fortalecer el proceso enseñanza aprendizaje EDUMECENTRO, 10(4):5471. Recuperado de https://www.medigraphic.com /pdfs/edumecentro/ed2018/ed184d.pdf

Rozo, A. (2010). Dimensión pedagógica de la Educación Virtual: Una reflexión pendiente. Recuperado de https://webcache.googleuserc ontent.com/search?q=cache: yyFBkBAzZGMJ:https://revist as.pedagogica.edu.co/index. php/PYS/article/download/74 2/718/+\&cd=9\&hl=es$419 \& \mathrm{ct}=\mathrm{clnk} \& \mathrm{gl}=\mathrm{ec}$

Sandoval, I, Molina, M, Cagua, B, Patricio, J. (2018). El video Educativo Como Estrategia Metodológica en la Enseñanza Universitaria. 16th LACCEI International Multi-Conference for Engineering, Education, and Technology: "Innovation in Education and Inclusion", 1921 July 2018, Lima, Perú. Recuperado 
https://www.researchgate.net/ publication/327710151_El_Vi deo_Educativo_Como_Estrat egia_Metodologica_en_la_En senanza_Universitaria/link/5b b2b887a6fdccd3cb813ba2/do wnload

Sierra-Llorente, J. G., PalmezanoCórdoba, Y. A., \& RomeroMora, B. S. (2018). CAUSAS QUE DETERMINAN LAS DIFICULTADES DE LA INCORPORACIÓN DE LAS TIC EN LAS AULAS DE CLASES. Revista Panorama, 12(22), 32 - 41. doi: http://dx.doi.org/10.15765/pnr m.v12i22.1064.

Silva, J. (2017). Un modelo pedagógico virtual centrado en las E-actividades. Revista de Educación a Distancia, 53 (10). 21-03. DOI: http://dx.doi.org/10.6018/red/ $53 / 10$

Unesco/lesalc. (2020). COVID-19 y educación superior: De los efectos inmediatos al día después. Recuperado de http://www.iesalc.unesco.org/ wpcontent/uploads/2020/05/CO VID-19-ES-130520.pdf

Vicentini, I. (2020). COVID-19: impacto en la educación y respuesta de la política públicas. Recuperado de https://publications.iadb.org/e s/la-educacion-superior-entiempos-de-covid-19-aportes- de-la-segunda-reunion-del-

dialogo-virtual-con 